\title{
Fungal conservation and ancient Egyptians- Part 2: A spiritual heritage of a look
}

\author{
Shafie $\mathbf{F R}^{*}$ \\ Department of Visual Arts, Faculty of Art and Architecture, Guilan University, Iran.
}

Shafie FR 2019 - Fungal conservation and ancient Egyptians- Part 2: A spiritual heritage of a look. Microbial Biosystems 4(1), 12-21.

\begin{abstract}
Egypt is the cradle of civilization and mycology. Drawing fungi is one of the joys of mycology, and something that anybody can try. Art has been a part of mycology since earliest times and published in many international journals and books. Line drawings are essential for showing details of fungal structure, whether in a field guide or fungal monographs. Ancient Egyptians produced a number of hieroglyphic depictions of psychedelic mushrooms on temples and through hieroglyphic texts throughout the country. A new attractive tool to raise national and international awareness on fungal conservation is drawing of fungi in many attractive portraits as ancient Egyptians documented them everywhere. In this series of paintings I participated with these portraits to attract young mycologists in MENA and to apply a new tool for conserving fungi.
\end{abstract}

Keywords - Egypt, fungi, cradle of mycology, Arab Society for Fungal Conservation.

\section{Introduction}

Scientists have unearthed fossilized fungi dating back up to one billion years, in a discovery that could reshape our understanding of how life on land evolved. For decades, the earliest known fungi (organisms such as mushrooms, mold and yeast) was thought to have appeared on earth around half a billion years ago. But recent fossil specimens unearthed in Canada and analysed using the latest dating technology appear to push back fungi's arrival to the earliest reaches of life on land.

Art is an essential aspect of any civilization. Once the basic human needs have been taken care of such as food, shelter, some form of community law, and a religious belief, cultures begin producing artwork, and often all of these developments occur more or less simultaneously. This process began in the Predynastic Period in Egypt through images of animals, human beings, and supernatural figures inscribed on rock walls. These early images were crude in comparison to later developments but still express an important value of Egyptian cultural consciousness: balance.

Egyptian society was based on the concept of harmony known as ma'at which had come into being at the dawn of creation and sustained the universe. All Egyptian art is based on perfect balance because it reflects the ideal world of the gods. The same way these gods provided all good gifts for humanity, so the artwork was imagined and created to provide a use. Egyptian art was always first and foremost functional.

This series of paintings focuses on the relationship between fungi and ancient Egyptian from my point of view to enhance mycologists, amateur mycologists and young generation to conserve fungi. 


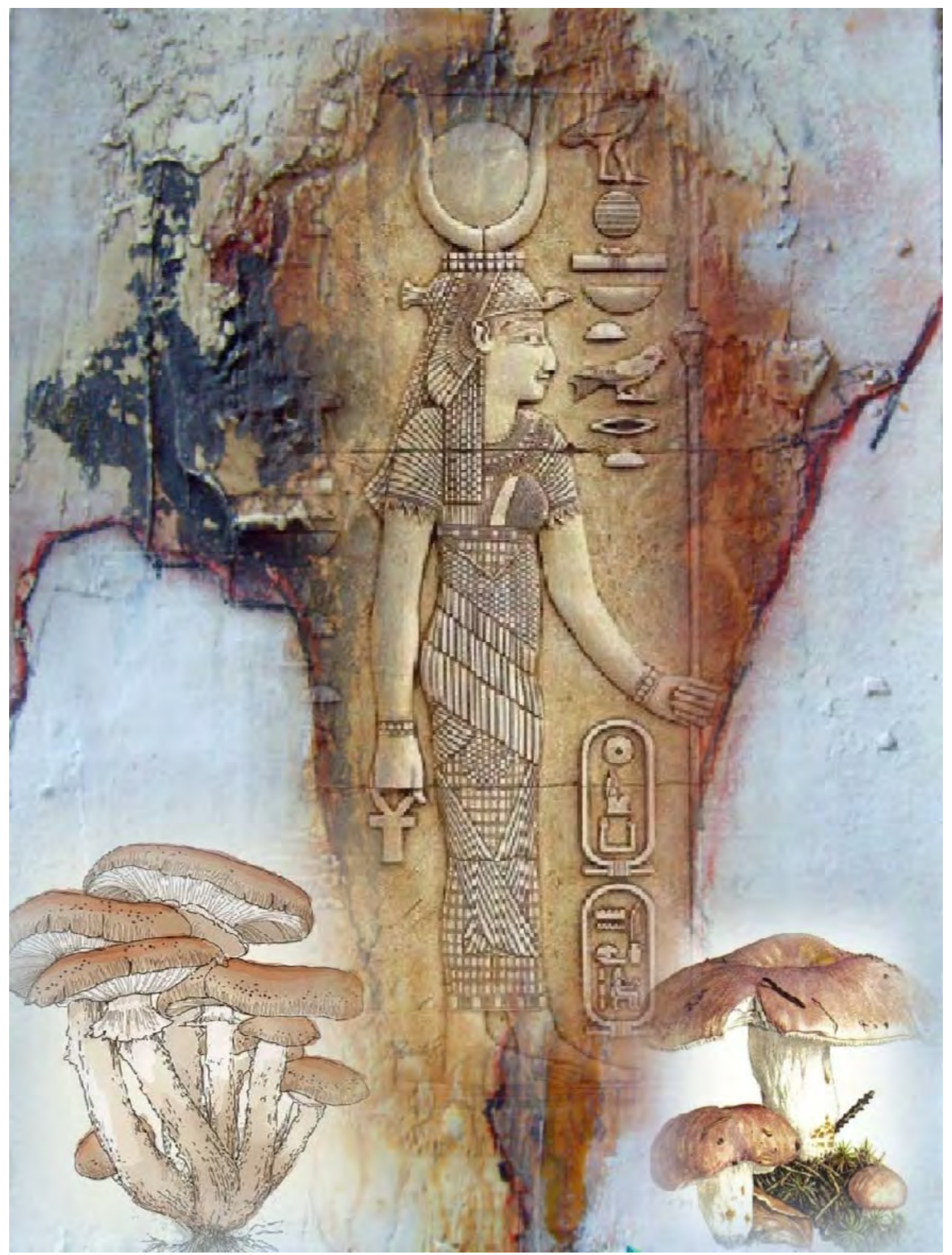

Technique: Mixed media on the wood.

Size: 50 x $70 \mathrm{~cm}$.

Date: 2019. 


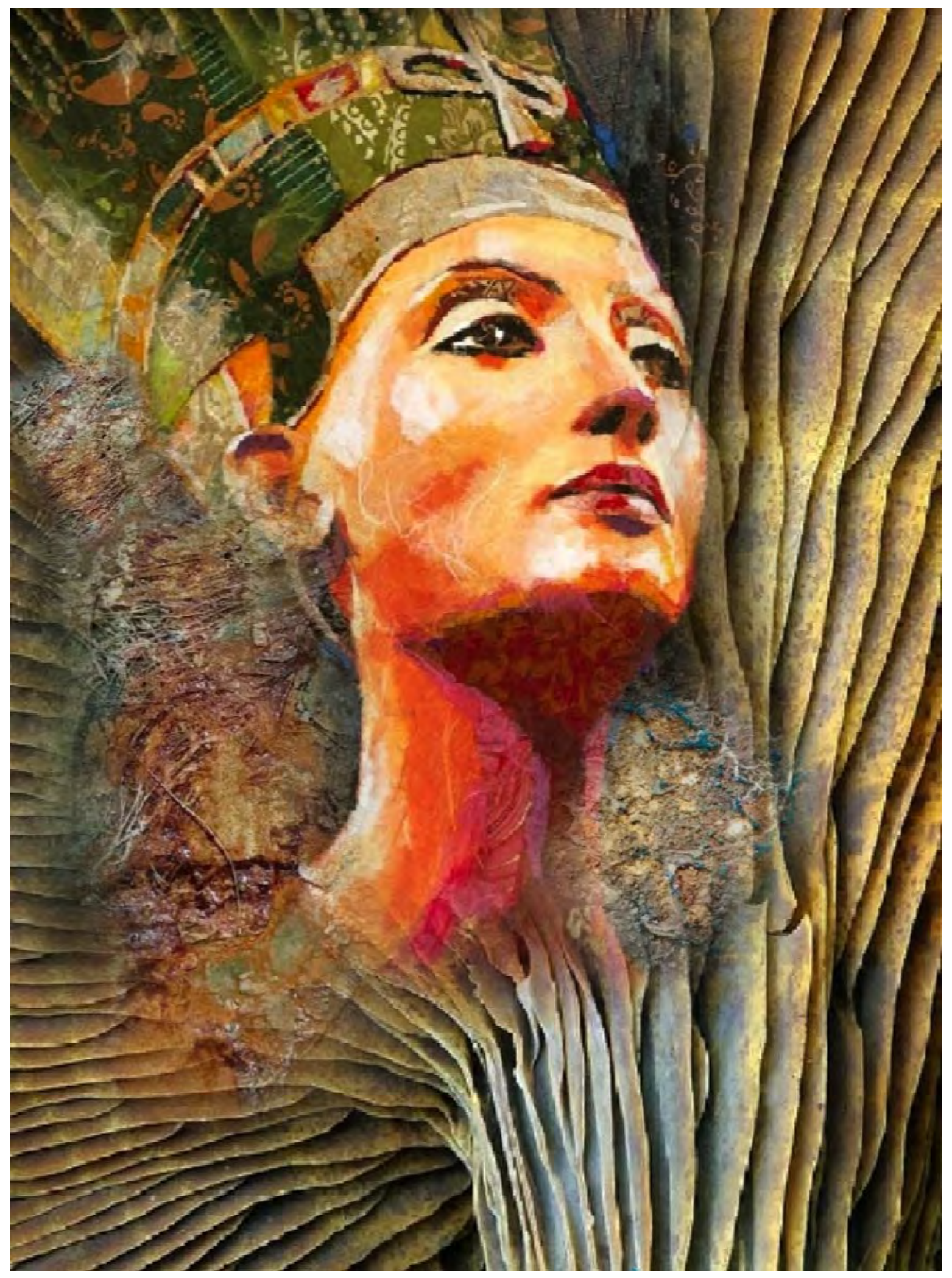

Technique: Mixed media and texture.

Size: 80 x $60 \mathrm{~cm}$.

Date: 2019. 


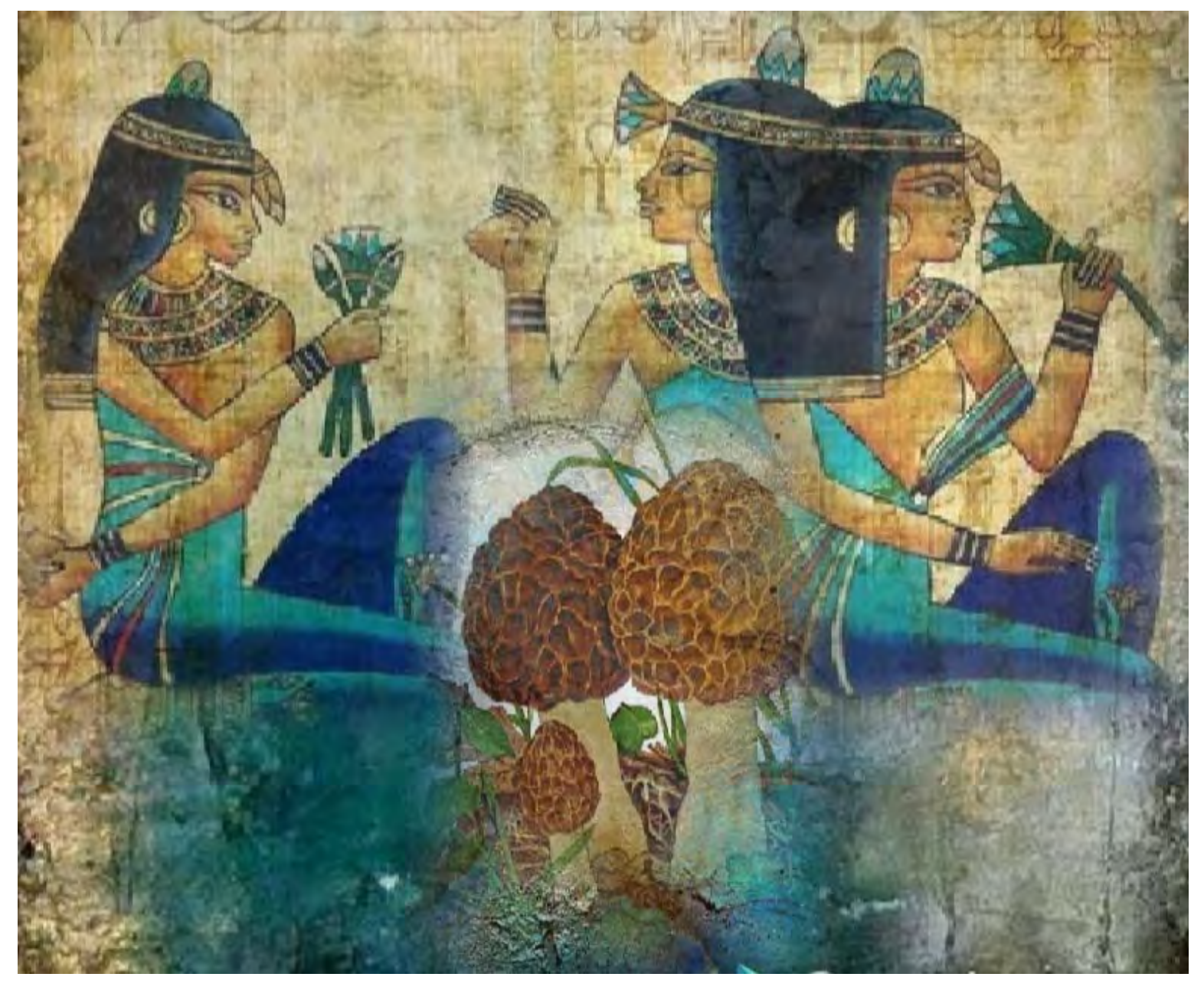

Technique: Mixed media.

Size: 50 x $70 \mathrm{~cm}$.

Date: 2019. 


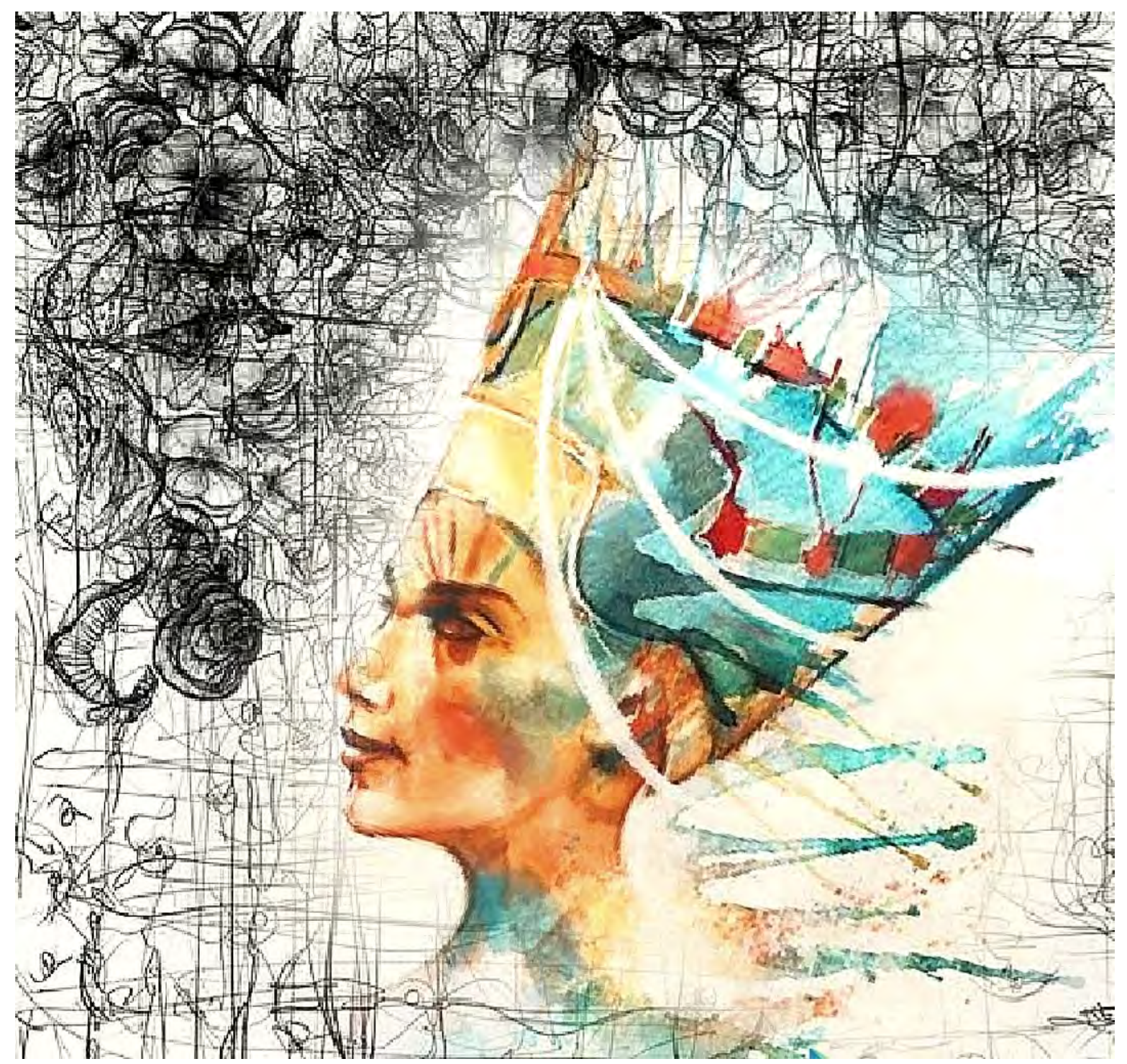

Technique: Mixed media and metal pen and ink. Size: 70 x $70 \mathrm{~cm}$.

Date: 2019. 


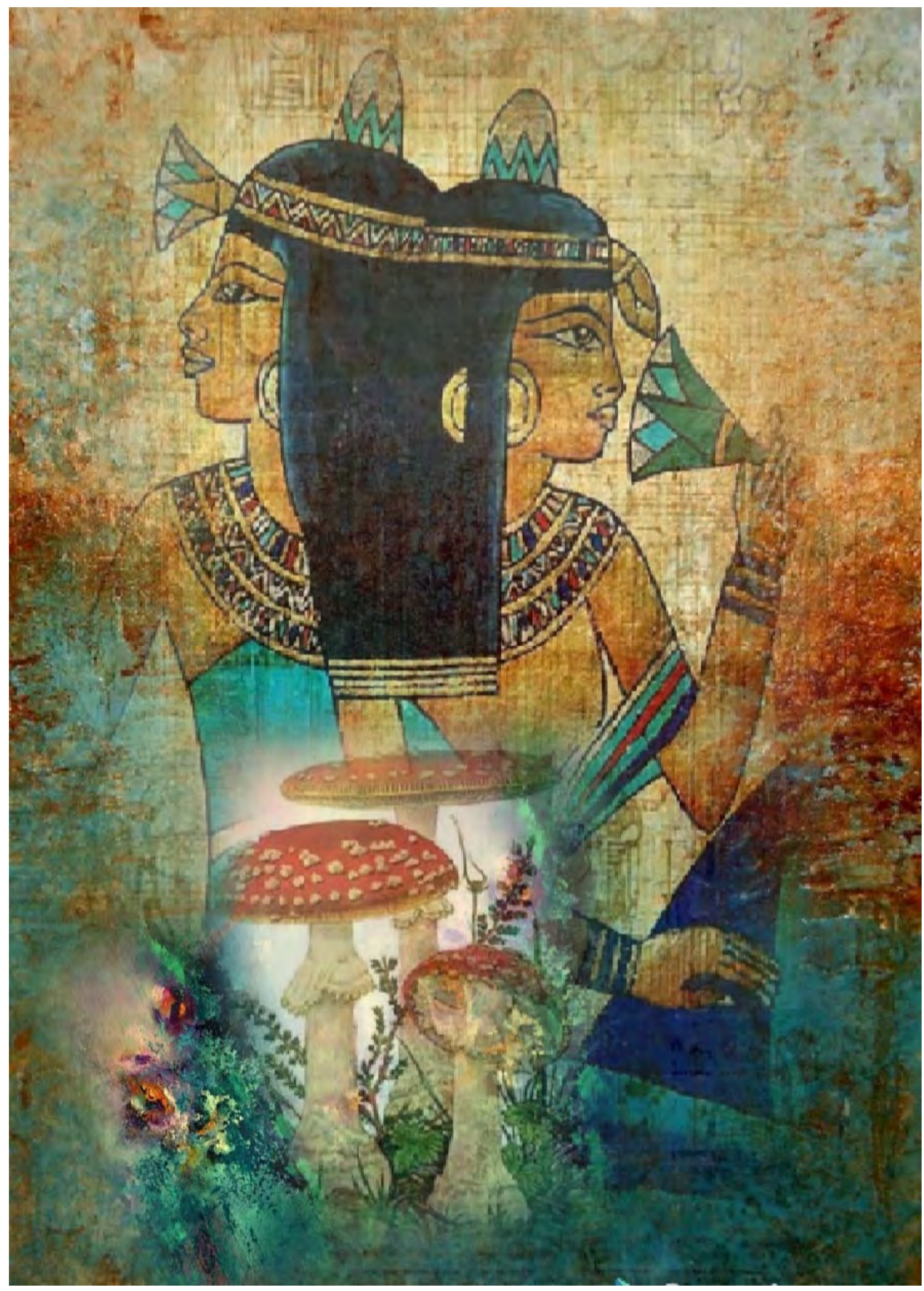

Technique: Mixed media and acrylic.

Size: 65 x $90 \mathrm{~cm}$.

Date: 2019. 


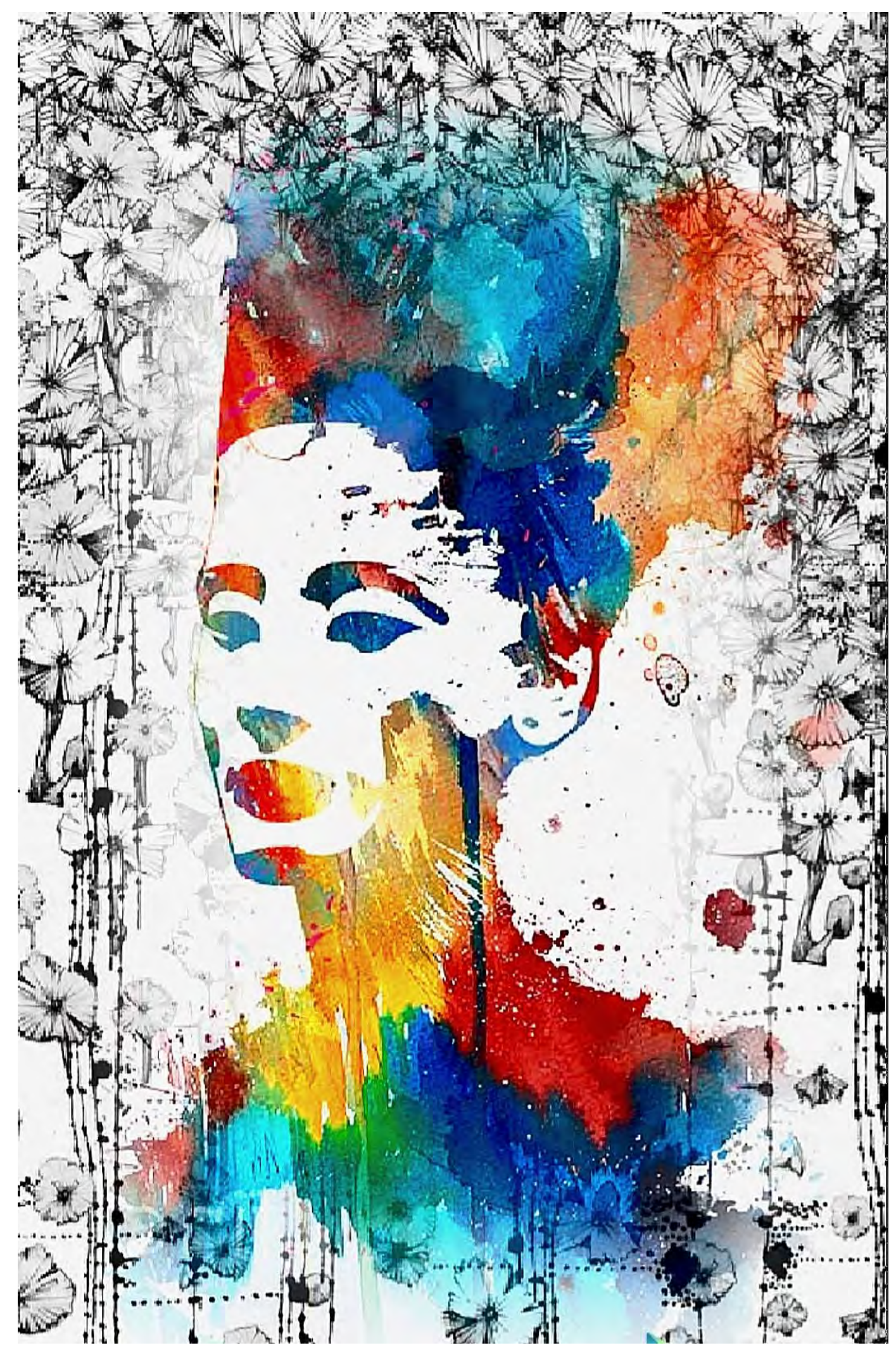

Technique: Ink, water colour and metal pen.

Size: 100 x $70 \mathrm{~cm}$.

Date: 2019. 


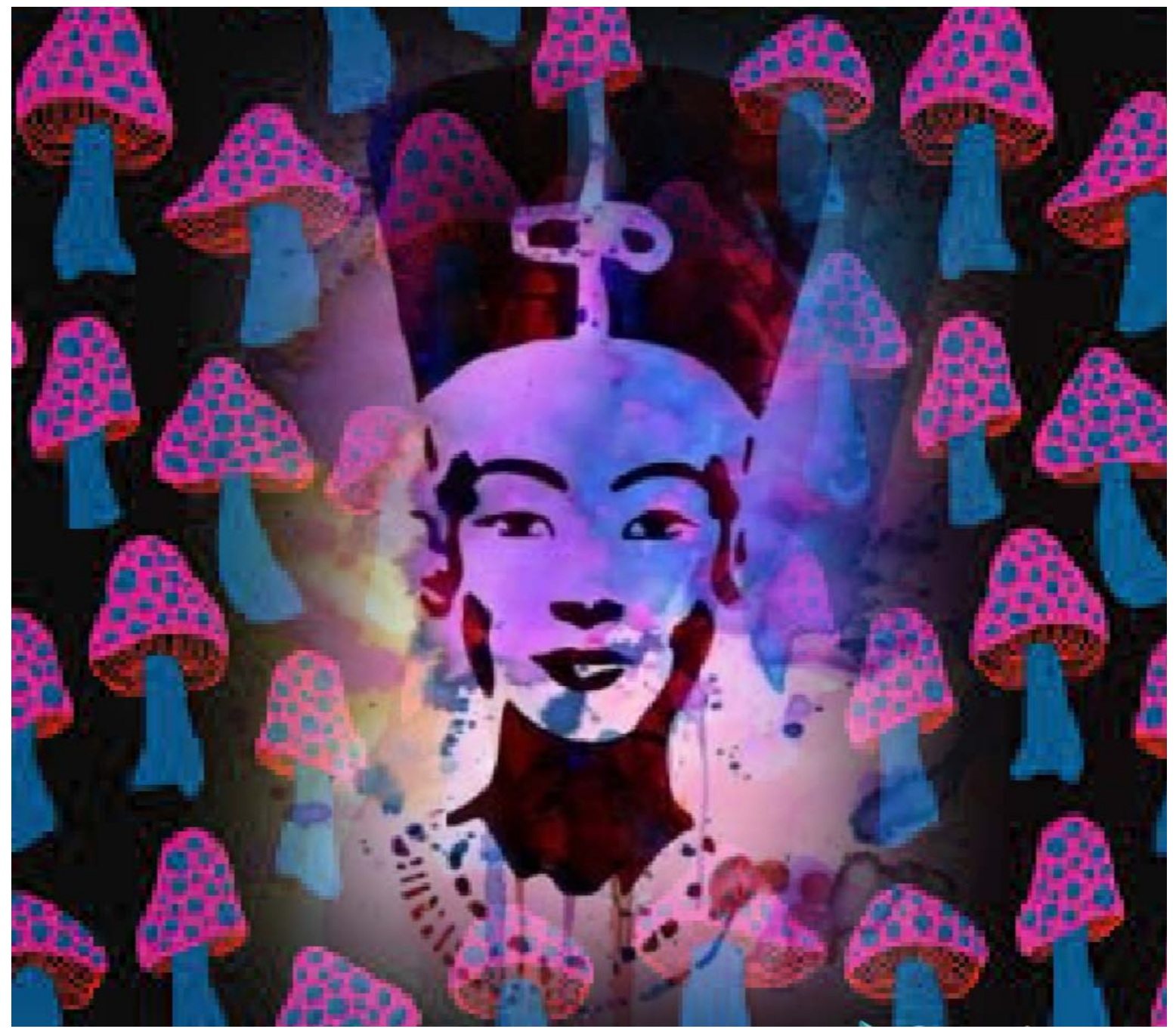

Technique: Mixed media and ink. Size: 60 x $60 \mathrm{~cm}$.

Date: 2019. 


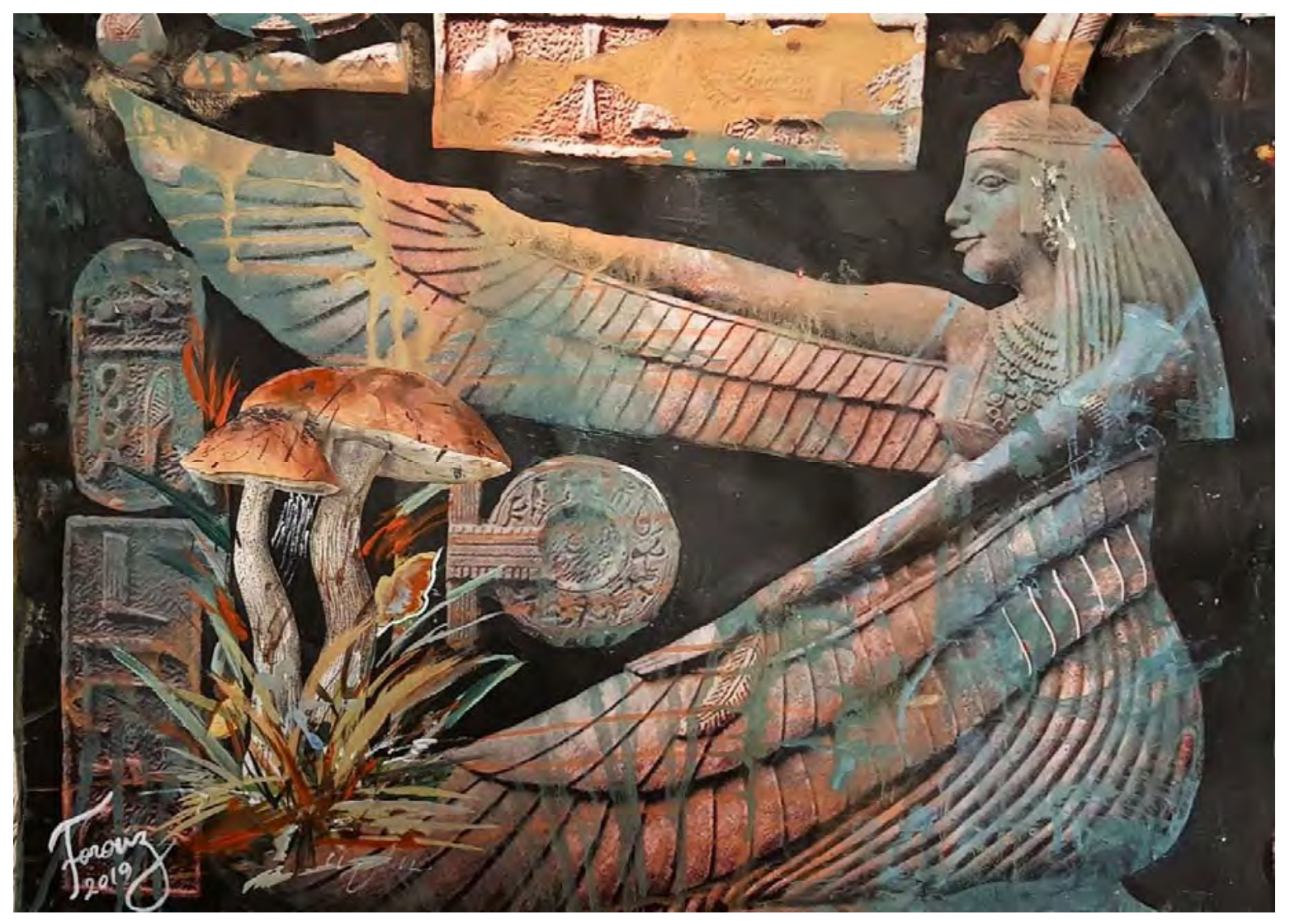

Technique: Mixed media.

Size: 60 x $80 \mathrm{~cm}$.

Date: 2019. 


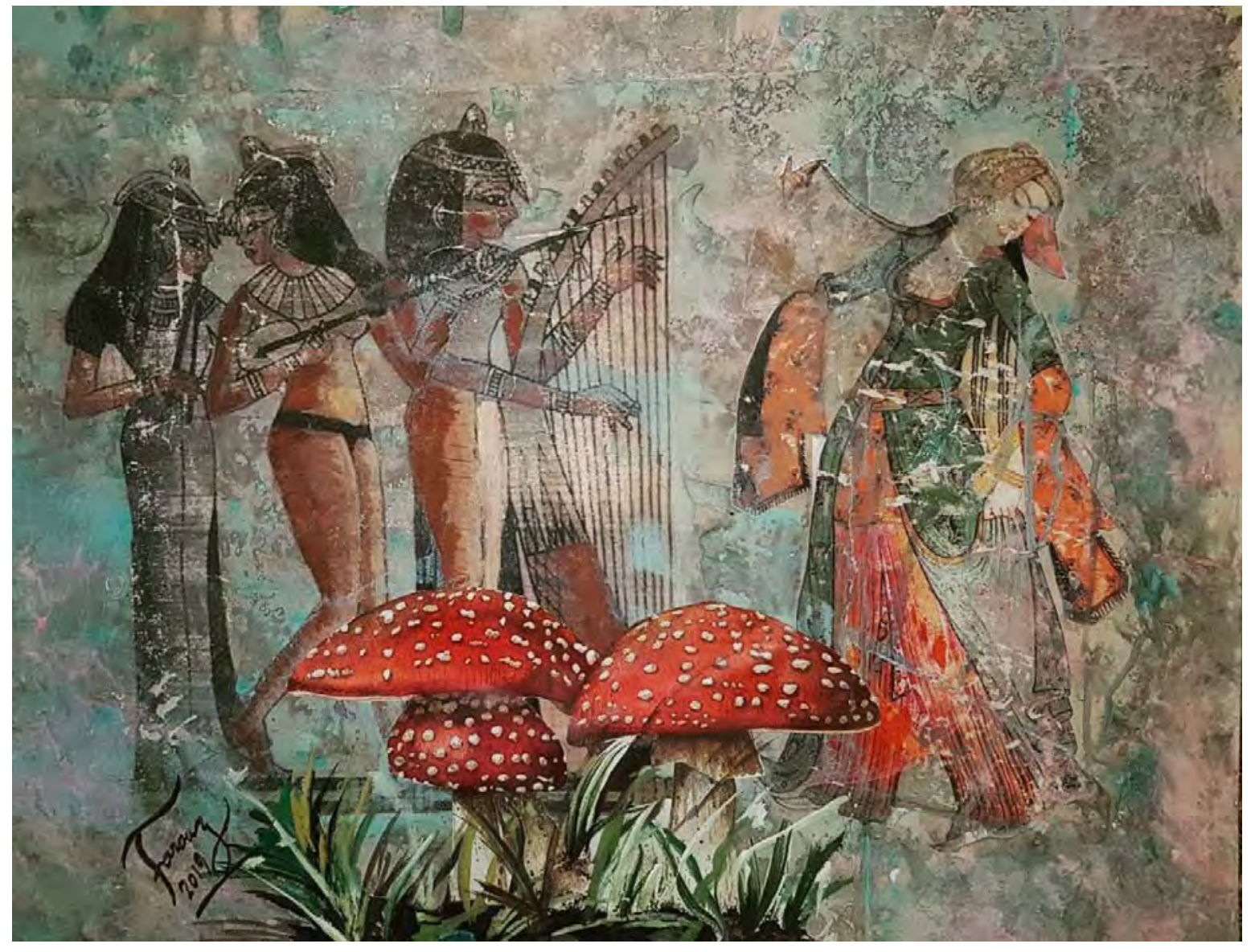

Technique: Mixed media.

Size: 70 x $90 \mathrm{~cm}$.

Date: 2019. 